The Impact of Large-Scale Surveys on Pulsating Star Research

ASP Conference Series, Vol. 203, 2000

L. Szabados \& D. W. Kurtz, eds.

\title{
On the Pulsation Properties of Red Supergiant Variables
}

\author{
Giuseppe Bono \\ Osservatorio Astronomico di Roma, Via Frascati 33, 00040 Monte \\ Porzio Catone, Italy \\ Nino Panagia \\ Space Telescope Science Institute, 3700 San Martin Drive, Baltimore, \\ $M D$ 21218, USA
}

\begin{abstract}
We present the preliminary results of a detailed theoretical investigation on the pulsation properties and modal stability of red supergiant (RSG) stars at solar chemical composition and for stellar masses ranging from 10 to $20 M_{\odot}$. We find that the main parameter governing their pulsation behavior is the effective temperature, and indeed when moving from the blue edge toward lower effective temperatures the models show an increase in the pulsation amplitudes. For temperatures cooler than $3800 \mathrm{~K}$ the pulsation amplitudes are still large but the radial displacements become aperiodic.
\end{abstract}

\section{Introduction}

There is considerable observational evidence that core-collapse supernovae (SN) undergo, 3000-5000 d after the explosion, substantial changes in the radio brightness curves. The change of the spectral index strongly suggests a variation in the density of the circumstellar material (CSM) and in particular in the canonical $r^{-2}$ law expected for pre-SN winds characterized by constant mass loss rates and expansion velocities (Montes et al. 1998; Chu et al. 1999, and references therein). The time scale brought out by radio measurements for variations in the radio fluxes $(\approx 10 \mathrm{yr}$ ) implies substantial changes in the pre-SN wind properties approximately $10,000 \mathrm{yr}$ before explosion. In fact, current data support the evidence that the SN shock front velocity is typically of the order of $10,000 \mathrm{~km} \mathrm{~s}^{-1}$, while the wind velocity of the $S N$ progenitor is roughly equal to $10 \mathrm{~km} \mathrm{~s}^{-1}$. The radio emission is governed by the mass loss rate to wind velocity ratio, $\dot{M} / w$, and therefore current radio fluctuations imply a variation of at least a factor of two in one or both these quantities $10^{4} \mathrm{yr}$ before the SN explosion. On the basis of these firm empirical facts it has been suggested that the occurrence of such a phenomenon cannot be caused neither by $\mathrm{H}$ and He burning evolutionary phases, nor by $\mathrm{C}$ burning phases, since the former ones are substantially longer, whereas the latter are much shorter (Chieffi, Limongi, \& Straniero 1999) than the typical time scale suggested by radio observations. The observational scenario on CSM properties is further enriched by optical spectroscopic measurements of emission lines which suggest the presence of slowly expanding shells with velocities 
ranging from 100 to $700 \mathrm{~km} \mathrm{~s}^{-1}$ (Ryder et al. 1993; Benetti et al. 1999, and references therein), thus strengthening the occurrence of shell ejections and/or compressed wind episodes during the pre-SN evolution.

In order to explain the appearance of such phenomena it has been recently suggested by Heber et al. (1997, hereinafter H97), on the basis of both linear and nonlinear pulsation models, that RSG stars are pulsationally unstable. In particular, they found evidence that for periods approaching the Kelvin-Helmholtz time scale these stars present large luminosity amplitudes, which in turn trigger a strong enhancement in the mass loss rate before they explode as supernovae. According to these authors this pulsation behavior should take place during the last few $10^{4}$ yr preceding the core collapse, due to the large increase in the luminosity to mass ratio experienced by RSG stars during these evolutionary phases. However, the nonlinear calculations performed by $\mathrm{H} 97$ were hampered by the fact that the hydrodynamic code they adopted could not properly handle pulsation destabilizations characterized both by small growth rates due to numerical damping and by large pulsation amplitudes due to the formation and propagation of strong shock waves during the approach to limit cycle stability. At the same time, H97 pointed out that the main theoretical drawback in dealing with the dynamical instabilities of RSG variables is the coupling between convection and pulsation. In fact, even though they constructed the linear models by assuming that the convective flux is frozen in, and the nonlinear ones by assuming that it is instantaneously adjusted, these treatments cannot account for the driving and/or quenching effects caused by the interaction between pulsation and convection.

These are the main reasons why only few theoretical investigations have been devoted to the pulsation behavior of RSG variables and date back to the seminal papers by Stothers \& Leung (1971) and by Fox \& Wood (1982) who provided a detailed analysis of evolutionary and pulsational properties of Galactic and Magellanic RSG variables. More recently Li \& Gong (1994), by comparing in the period-luminosity plane theoretical predictions and observational data, suggested that RSG variables in the Large Magellanic Cloud are fundamental mode pulsators. However, since this investigation was based on linear models, no firm conclusion could be reached concerning the actual properties of these objects and, in particular, on pulsation amplitudes. Finally, we mention that even though homogeneous photometric databases on RSG variables are not available in the literature, recent microlensing experiments should provide accurate constraints on the pulsation behavior of these objects (Wood et al. 1999).

\section{Theoretical Framework and Main Results}

The theoretical framework adopted for constructing both linear and nonlinear models of high-mass radial variables was already described in previous papers (Bono \& Stellingwerf 1994; Bono, Caputo, \& Marconi 1998; Bono, Marconi \& Stellingwerf 1999). In order to provide a detailed analysis of RSG pulsation properties during both $\mathrm{H}$ and $\mathrm{He}$ burning phases we constructed several sequences of models at fixed chemical composition $(Y=0.28, Z=0.02)$ which cover a wide range of stellar masses $\left(10 \leq M / M_{\odot} \leq 20\right)$. Moreover, since we are interested in mapping the properties of RSG stars not only during the central He burning but 
also during the $\mathrm{H}$ shell burning and up to the central He exhaustion, both the luminosities and the effective temperature values were selected directly along the evolutionary tracks. The evolutionary calculations were performed at fixed mass - i.e. no mass-loss - and by neglecting the effects of both convective core overshooting and rotation.

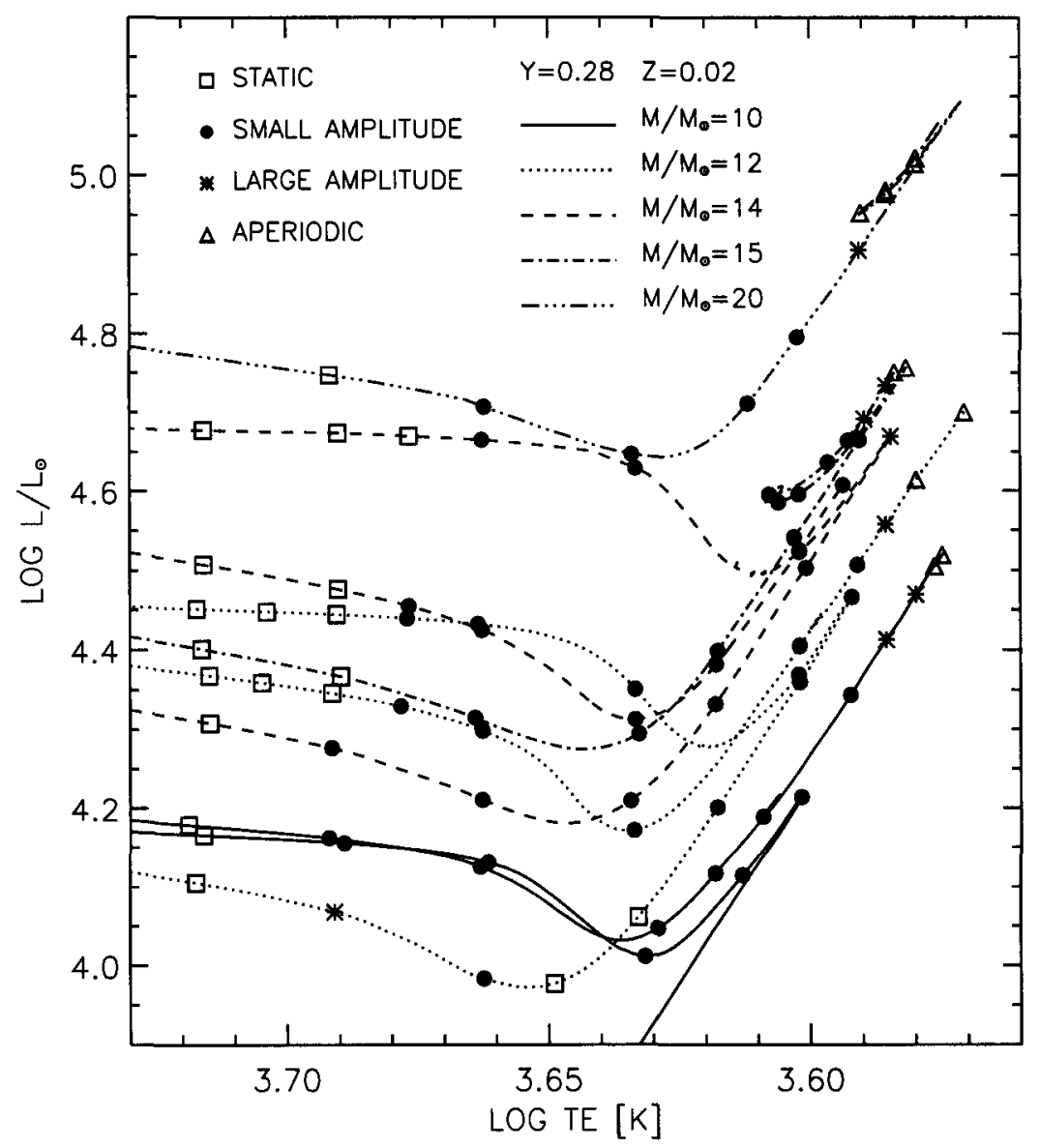

Figure 1. Evolutionary tracks and pulsation models in the HR diagram. See text for further details.

The input physics and physical assumptions adopted for constructing evolutionary and pulsational models will be described in detail in a forthcoming paper (Panagia \& Bono 2000). Fig. 1 shows the location in the HR diagram of both evolutionary tracks and pulsation models we constructed. The pulsation models characterized by different limiting amplitude behavior are plotted by adopting different symbols. The squares refer to models which are pulsationally stable (i.e. after the initial perturbation the radial motions decay and the structure approaches once again the static configuration), while filled circles and asterisks refer to models which show small and large $\left(A_{\mathrm{bol}}>1 \mathrm{mag}\right)$ pulsa- 
tion amplitudes together with a periodic behavior (stable limit cycle). Triangles display the models which present large pulsation amplitudes but aperiodic radial displacements (unstable limit cycle). The behavior of pulsation properties discloses several interesting features: 1) For effective temperatures lower than approximately $5100 \mathrm{~K}$ high-mass models are, with few exceptions, pulsationally unstable in the fundamental mode both during $\mathrm{H}$ shell and He burning phases. 2) The pulsational behavior is mainly governed by the effective temperature and, to a lesser extent, by the luminosity. In fact, the transition from small to large pulsation amplitudes $\left(T_{\mathrm{e}} \approx 3900 \mathrm{~K}\right)$ and from periodic to aperiodic behavior $\left(T_{\mathrm{e}} \leq 3800 \mathrm{~K}\right)$ take place roughly at constant temperature. 3) The region in which the models attain small amplitudes is the natural extension of the classical Cepheid instability strip, thus confirming the empirical evidence originally brought out by Eichendorf \& Reipurth (1979) and more recently by Kienzle et al. (1998), as well as the theoretical prediction by Soukup \& Cox (1996).

The RSGs are among the most luminous stars in the sky, and therefore they can be easily identified in spiral and dwarf galaxies located at large distances. The results of our investigation support the evidence that low amplitude RSG variables could also be good standard candles.

\section{References}

Benetti, S., Turatto, M., Cappellaro, E., Danziger, I. J., \& Mazzali, P. 1999, MNRAS, 305, 811

Bono, G., Caputo, F., \& Marconi, M. 1998, ApJ, 497, L43

Bono, G., Marconi, M., \& Stellingwerf, R. F. 1999, ApJS, 122, 167

Bono, G. \& Stellingwerf, R. F. 1994, ApJS, 93, 233

Chieffi, A., Limongi, M., \& Straniero, O. 1998, ApJ, 502, 737

Chu, Y-H., Caulet, A., Montes, M. J., Panagia, N., Van Dyck, S. D., \& Weiler, K. W. 1999, ApJ, 512, L51

Eichendorf, W. \& Reipurth, B. 1979, A\&A, 77, 227

Fox, M. W. \& Wood, P. R. 1982, ApJ, 259, 198

Heber, A., Jeannin, L., Langer, N., \& Baraffe, I. 1997, A\&A, 327, 224 [H97]

Kienzle, F., Burki, G., Burnet, M., \& Meynet, G. 1998, A\&A, 337, 779

Li, Y. \& Gong, Z. G. 1994, A\&A, 289, 449

Montes, M. J., Van Dyck, S. D., Weiler, K. W., Sramek, R. A., \& Panagia, N. 1998, ApJ, 506, 874

Panagia, N. \& Bono, G. 2000, in The Largest Explosions Since the Big Bang: Supernovae and Gamma Ray Bursts, ed. M. Livio, F. Boffi, \& N. Panagia (Cambridge: Cambridge University Press), in press

Ryder, S., Staveley-Smith, L., Dopita, M., et al. 1993, ApJ, 416, 167

Soukup, M. S. \& Cox, A. N. 1996, BAAS, 28, 917

Stothers, R. \& Leung, K. C. 1971, A\&A, 10, 290

Wood, P. R., Alcock, C., Allsman, R. A., et al. (The Macho Collaboration) 1999, in IAU Symp. 191, AGB Stars (San Francisco: ASP), in press 